\title{
Betacarotenemia with isolated nose involvement in a child: a case report
}

\author{
Assoc. Prof. Burcu Kayhan-Tetik, M.D. ${ }^{a}$ and Nilgün Çaylan, M.D. ${ }^{b}$
}

\begin{abstract}
Carotenemia is generally benign and usually caused by excessive intake of beta-carotene rich food. This clinical presentation can occur in any age group but frequently occurs in children. We report a rare case of carotenemia with an isolated involvement of the nose and presented in order to remind carotenemia in the differential diagnosis of jaundice. Carotenemia is often easily recognized and dealt with by family medicine and pediatricians who may potentially get involved in the management of these cases. It is also important to know and recognize carotenemia in terms of informing families and preventing unnecessary tests.

Key words: carotenemia, beta carotene, jaundice, vitamin A.
\end{abstract}

http:/ / dx.doi.org/10.5546/ aap.2019.eng.e502

To cite: Kayhan-Tetik B, Çaylan N. Betacarotenemia with isolated nose involvement in a child: a case report. Arch Argent Pediatr 2019;117(5):e502-e504.

\section{INTRODUCTION}

Carotenemia is a clinical entity characterized by yellowish discoloration of the skin, resulting from increase in serum beta-carotene levels and carotene deposition mainly in the stratum corneum. Although beta carotenemia have been considered to be a rare disease, its prevalence is not known. However, in Priyadarshani's study conducted on 615 children the prevalence was found the to be $2 \% .{ }^{1}$ This clinical presentation

a. Department of Family Medicine, Inonu University Medical Faculty, Malatya.

b. Public Health Institution, Child and Adolescent Health Department, Ankara, Turkey.

E-mail address:

Burcu Kayhan-Tetik: drburcukayhan@hotmail.com

Funding: None.

Conflict of interest: None.

Received: 8-6-2018

Accepted: 2-25-2019 can occur in any age group but frequently occurs in children. ${ }^{2}$ Carotenemia is generally benign and usually caused by excessive intake of beta-carotene rich food. However, it is rarely associated with a few serious metabolic disorders and inborn enzyme defects that metabolize carotenoids. $^{3}$

We present this case because carotenemia is rarely seen isolated on the tip of the nose and it should be taken into consideration in the differential diagnosis of jaundice.

\section{CASE}

A 3-years old, previously healthy girl presented with a one-month history of yellowishorange discoloration of the nose. Clinical history revealed that this color first occurred when she was 3 years old and repeated 5 times. She did not have any constitutional symptoms and her urine and stool colors were normal. In the family history there was no one in the family with similar symptoms. Physical examination revealed a yellowish-orange discoloration of her nose wings and tip of the nose (Figure 1). The palms, soles, other parts of skin and sclera of her eyes were normally colored. The rest of the physical examination was within normal limits and her growth and development were normal. Laboratory tests revealed a normal complete blood count, renal function tests, thyroid function tests, and lipid profile. Liver function tests, serum bilirubin and viral serology for hepatitis A and B were within normal limits. Serum beta-carotene was markedly elevated at $368 \mu \mathrm{mol} / \mathrm{L}$ (reference range: $60-200 \mu \mathrm{mol} / \mathrm{L}$ ). Serum vitamin A level was normal at $72 \mu \mathrm{mol} / \mathrm{L}$ (normal range: 30 $80 \mu \mathrm{mol} / \mathrm{L}$ ). When the story was detailed, we learned that she ate excessive amounts of carrot, orange and mandarin on the diet, recently and the yellowish color on the skin appeared after that. She was diagnosed with carotenemia and her parents have been suggested to remove carrots, oranges and mandarin, which are beta-carotene rich foods, from the diet. In the follow up, it was seen that the patient conformed the diet and the yellowish color change in her nose completely disappeared (Figure 2). 


\section{DISCUSSION}

Carotenoids are organic compounds found in many red, orange or yellow colored fruits, vegetables and in green-leafy vegetables. Foods with a high concentration of carotenoids include carrots, apricots, orange, mandarin, mangoes, sweet potatoes, kale, melon, papaya and other green leafy vegetables. ${ }^{2}$ Of the carotenoids, particularly beta-carotene, is the most important one and gets absorbed from the small intestine. Following absorption, it is converted to two molecules of retinol, a vitamin A metabolite in the mucosal cells of the small intestine by the beta-carotene 15,15 'dioxygenase enzyme, then it enters the lymphatic system. ${ }^{4}$ Approximately $10 \%$ of the ingested beta-carotene enters the portal circulation unchanged. Beta-carotene is transported in the serum by lipoproteins and eliminated primarily through the colon, and the epidermis via sweat and sebaceous material. ${ }^{5}$

Carotenoids begin to accumulate in the epidermis about 2 weeks after serum levels achieve equilibrium. ${ }^{6}$ Generally, the yellow pigmentation of beta-carotene appears when its concentrations in the serum exceed $250 \mathrm{mg} / \mathrm{dL}^{2}$ The stratum corneum has a high lipid content which has an affinity for carotene, and so the carotene pigment is concentrated in the stratum corneum. ${ }^{2}$ The yellowish discoloration of the skin that characterizes carotenemia is commonly manifests in areas of thickened stratum corneum and in areas with an abundance of sweat glands. The pigmentation is usually appears on the palms and soles, nasolabial folds, tip of the nose, forehead, chin, knuckles, and behind the ears and may sometimes appear on the palate. ${ }^{2,5}$ Typically, scleral involvement is not seen in carotenemia cases. In our case, only wings and tip of the nose were involved. In other areas, especially in the

FIGURE 1. Yellowish discoloration in the nose wings and tip of nose

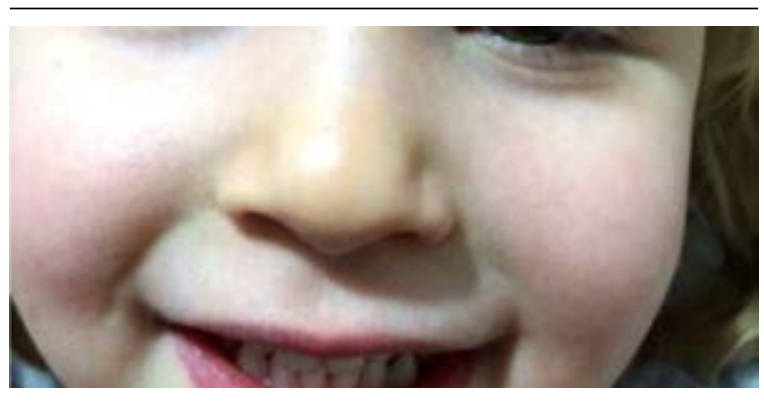

palms and soles, there was no involvement and sclera was normal.

When diagnosis of carotenemia is considered, first step is to exclude jaundice. While scleral icterus is an early clinical sign of jaundice, carotenoids do not accumulate in the sclera; the presence of clear sclera is an important clinical finding in carotenemia diagnosis. ${ }^{6}$ In addition, in the presence of a disease that causes jaundice, color change in the skin is often accompanied by other findings such as fever, pruritus, weakness, vomiting, color change in stool, and liver enlargement. In addition, Gilbert syndrome, a congenital bilirubin metabolism disorder, is also one of the diseases that may be confused with carotenemia. In the differential diagnosis, especially the Gilbert syndrome is diagnosed by increased indirect bilirubin in the blood together with fatigue and stress. Differential diagnosis can be made by the absence of increased indirect bilirubin in the blood when is present. Once jaundice and Gilbert syndrome have been ruled out, it should be questioned especially, whether there is an excessive amount of betacarotene-rich food consumption. In our case, excessive consumption of carotene containing fruits was mentioned. Cases of carotenemia have also been reported with nutrient supplements and processed foods as well as natural foods containing carotenoids. ${ }^{6,7}$ If diet is found not to be responsible for the carotenemia, the physician should consider conditions including diabetes mellitus, hypothyroidism, anorexia nervosa, liver and kidney disease. ${ }^{3}$

Generally, differential diagnosis can be performed easily by a good history, physical examination and simple blood tests. Rarely, carotenaemia is associated with inborn error of metabolism result from partial or complete

FIGURE 2. Yellowish discoloration was disappeared after the dietary restriction 
deficiency of 15-15'-dioxygenase. This enzyme defect may be familial and leads to accumulation of beta-carotene and low to normal vitamin $\mathrm{A}$ levels. ${ }^{8,9}$ In this case it has been thought that betacarotenemia is largely dependent on diet rather than familial transition because the level of vitamin A was normal, no similar clinical findings were found in any family member before, food containing betacarotene has been consumed excessively, and the symptoms responded to dietary restriction. In addition, diseases that can lead to jaundice are easily excluded due to the normal history, physical examination, and laboratory findings.

Yellowish skin may also be emerged by lycopenemia, riboflavinemia, percutaneous absorption or ingestion of some drugs and chemicals including quinacrine hydrochloride, saffron, 2,4-dinitrophenol, and picric acid and they should be considered in differential diagnosis of carotenemia. ${ }^{3,9}$

\section{CONCLUSION}

Carotenemia is generally a benign condition and excessive intake of carotenoids is not associated with vitamin A toxicity. Carotenemia is often easily recognized and adressed by family medicine and pediatricians who may potentially get involved in the management of these cases. It is also important to know and recognize carotenemia for informing families and preventing unnecessary tests.

\section{REFERENCES}

1. Priyadarshani AMB. Insights of hypercarotenaemia: A brief review. Clin Nutr ESPEN. 2018; 23:19-24.

2. Maharshak N, Shapiro J, Trau H. Carotenoderma-a review of the current literature. Int J Dermatol. 2003; 42(3):178-81.

3. Lascari AD. Carotenemia: A review. Clin Pediatr (Phila).1981; 20(1):25-9.

4. Lakshman MR, Okoh C. Enzymaticconversion of all-trans beta-caroteneintoretinal. Methods Enzymol. 1993; 214:256-69.

5. Prince MR, Frisoli JK. Beta-caroteneaccumulation in serum and skin. Am J Clin Nutr. 1993; 57(2):175-81.

6. Karthik SV, Campbell-Davidson D, Isherwood D. Carotenemia in infancy and its association with prevalent feeding practices. Pediatr Dermatol. 2006; 23(6):571-3.

7. Takita Y, Ichimiya M, Hamamoto Y, Muto M. A case of carotenemia associated with ingestion of nutrient supplements. J Dermatol. 2006; 33(2):132-4.

8. Chattopadhyay M, Pramanik R, McGrath JA, Burrows NP. Familial carotenaemia and carotenoderma. Clin Exp Dermatol. 2014; 39(6):771-2.

9. Chattopadhyay M, Pramanik R, McGrath JA, Burrows NP. Familial carotenaemia and carotenoderma. Clin Exp Dermatol. 2014; 39(6):771-2. 